\title{
Grey matter volumes in treatment naïve vs. chronically treated children with attention deficit/hyperactivity disorder: a combined approach
}

\author{
Thomas Villemonteix ${ }^{a}$ * , Stéphane A. De Brito ${ }^{b}$, Martin Kavec ${ }^{c}$, \\ Danielle Balériaux ${ }^{c}$, Thierry Metens ${ }^{c}$, Hichem Slama ${ }^{\mathrm{d}, e, f}$, \\ Simon Baijot ${ }^{\mathrm{d}, \mathrm{f}}$, Alison Mary ${ }^{\mathrm{f}}$, Philippe Peigneux ${ }^{\mathrm{f}, \mathrm{g}}$, \\ Isabelle Massat ${ }^{a, f, g, h}$
}

\author{
aINSERM, U894, 2 ter rue d'Alésia, 75014 Paris, France \\ dUNESCOG - Research Unit in Cognitive Neurosciences, ULB, Belgium \\ 1070 Brussels, Belgium \\ ${ }^{f}$ UR2NF - Université Libre de Bruxelles (ULB), Belgium \\ ${ }^{\mathrm{g} U N I}$ - ULB Neurosciences Institute, ULB, Belgium \\ ${ }^{\mathrm{h}}$ National Fund of Scientific Research (FNRS), Belgium
}

${ }^{\mathrm{b} S}$ School of psychology, University of Birmingham, Edgbaston, Birmingham B15 2TTT

'Department of Radiology, Clinics of Magnetic Resonance, Erasme Hospital, Brussels, Belgium, UK

e Department of Clinical and Cognitive Neuropsychology, Erasme Hospital, 808 Lennik Street, CP601,

Received 26 December 2014; received in revised form 2 April 2015; accepted 10 April 2015

\section{KEYWORDS}

Attention deficit hyperactivity disorder (ADHD);

Structural MRI;

Voxel-based morpho-

metry;

Methylphenidate;

Nucleus accumbens

\begin{abstract}
Psychostimulants are the first-line treatment in attention deficit/hyperactivity disorder $(A D H D)$, but their effects on brain development remain poorly understood. In particular, previous structural magnetic resonance imaging (sMRI) studies only investigated treatment effects on grey matter (GM) volumes in selected regions of interest (ROls). In this study, voxelbased morphometry (VBM) was used to assess medication-related GM volume differences across the entire brain. Automated tracing measurements of selected ROls were also obtained. Three groups (77 participants aged 7-to-13 year old) underwent MRI scans and were compared: nevermedicated children with ADHD $(n=33)$, medicated (methylphenidate) children with ADHD $(n=20)$ and typically developing children (TD; $n=24)$. Optimised VBM was used to investigate regional GM volumes, controlling for age and gender. Automated tracing procedures were also used to assess the average volume of the caudate nucleus, the amygdala and the nucleus
\end{abstract}

*Correspondence to: Centre Psychiatrie et Neurosciences, team 1, 2 ter rue d'Alésia, 75014 Paris, France. Tel.: + 33661572037 ; fax: + 33145807293 .

E-mail address: t.villemonteix@gmail.com (T. Villemonteix). 
accumbens. When compared to both medicated children with ADHD and TD children, nevermedicated children with ADHD exhibited decreased GM volume in the insula and in the middle temporal gyrus. When compared to TD children, medicated children with ADHD had decreased GM volume in the middle frontal gyrus and in the precentral gyrus. Finally, ROI analyses revealed a significant association between duration of treatment and GM volume of the left nucleus accumbens in medicated children with ADHD. In conclusion, this study documents potential methylphenidate-related GM volume normalization and deviation in previously unexplored brain structures, and reports a positive association between treatment history and GM volume in the nucleus accumbens, a key region for reward-processing.

(c) 2015 Elsevier B.V. and ECNP. All rights reserved.

\section{Introduction}

Psychostimulants such as methylphenidate or amphetamines are the most commonly used psychotropic medications in children and adolescents (Zuvekas and Vitiello, 2012). In 2008, it was estimated that around 4 million children were treated with stimulants each day in the USA (Zuvekas and Vitiello, 2012). Methylphenidate, in particular, is the firstline treatment for attention deficit/hyperactivity disorder (ADHD) (Biederman and Faraone, 2005). Despite its widespread prescription, an ongoing debate exists regarding its long-term effects on the brain, especially regarding substance abuse liability (Kim et al., 2009; Volkow 2012). Methylphenidate indeed increases dopamine levels in the nucleus accumbens, a pharmacological effect underlying drug reward that may trigger the neuroadaptations in dopaminergic and glutamatergic signaling associated with addiction (Volkow, 2012). Due in part to their impulsivity, children and adolescent with ADHD are known to be at risk for the development of substance abuse (Levy et al., 2014). In 2006, a report indicated that more than 7 million people in the US had abused ADHD stimulants, and that as many as 750,000 teenagers may show signs of addiction (Kroutil et al., 2006).

In this context, neuroimaging techniques represent valuable tools to track down the potential effects of medication on the human brain. In the last two decades, several crosssectional structural magnetic brain imaging (sMRI) studies have compared volumes of specific brain regions between children with ADHD who were medication-naïve, children with ADHD who had been using methylphenidate, and typically developing (TD) children. An early study examined the grey matter (GM) volumes of the caudate nucleus, the cerebellum and the four major lobes using automated tracing procedures, and reported no significant effect of medication (Castellanos et al., 2002). Subsequent cross-sectional studies examined GM volumes of the caudate nucleus (SemrudClikeman et al., 2006; Sobel et al., 2010), the anterior cingulate cortex (ACC; Semrud-Clikeman et al., 2006), the cerebellum (Bledsoe et al., 2009), the thalamus (Ivanov et al., 2010), the putamen, the globus pallidus (Sobel et al., 2010) and the nucleus accumbens (Hoekzema et al., 2014). These studies generally reported normalizing effects of medication on GM volume deficits found in ADHD patients (Schweren et al., 2013), except for Hoekzema and colleagues who found substantial volumetric declines in the bilateral nucleus accumbens in medicated patients. However, analysis of longitudinal data suggested that this initial reduction was followed by a recovery of volume under maintained treatment (Hoekzema et al., 2014). Finally, in a recent longitudinal study, Shaw and colleagues examined the volumes of the caudate nucleus, the putamen and the globus pallidus in a large cohort of children with ADHD and TD children (Shaw et al., 2014). They reported no significant association between history of psychostimulant treatment and developmental trajectories.

Grey matter volumes are only one among different possible indicators of brain structure, along with cortical thickness, surface area and cortical gyrification (Winkler et al., 2009). One longitudinal study examined change in cortical thickness in medicated and never-medicated adolescents with ADHD when compared to TD adolescents, and found an excessive rate of cortical thinning in the right motor strip, the left middle/inferior frontal gyrus and the right parieto-occipital region in the never-medicated group only (Shaw et al., 2009). In a larger sample encompassing the participants recruited in this study, no significant differences in cortical thickness were detected at study entrance when comparing medicated and never-medicated children with $\mathrm{ADHD}$, except in a small region in the anterior temporal cortex (Shaw et al., 2006).

Taken together, these findings support the view that administration of methylphenidate is not detrimental to the development of the human brain (Schweren et al., 2013). However, this literature is characterized by several limitations. First, despite a preferential activation of prefrontal cortex catecholamine neurotransmission by methylphenidate (Spencer et al., 2012), none of these studies examined medication effects on GM volumes in frontal regions (only the volume of the entire frontal lobe was once examined (Castellanos et al., 2002)). Second, effect of methylphenidate on grey matter volume in the nucleus accumbens was only examined in children medicated for less than 13 months (Hoekzema et al., 2014). Longer treatment durations may be required to identify noticeable structural changes in this structure. Third, the above sMRI studies relied on lobar manual or automated region of interest (ROI) measurement methods. ROI methods yield a single value for the volume of the region examined, obtained after averaging signal over the ROI. This signal 
averaging can cause a dilution of the measure of the volume difference, especially when this difference is only present in a limited part of the ROI (Voormolen et al., 2010). Voxelbased morphometry (VBM) is an alternative automated approach that employs a general linear model framework to conduct mass voxel wise statistical tests throughout the brain, allowing it to be a particularly sensitive technique for detecting focal differences in GM volumes within all regions of the brain (Ashburner and Friston, 2000). VBM has been shown to outperform ROI methods when detecting focal differences in morphology (Voormolen et al., 2010). However, theoretically, ROI methods remain superior when between-group differences are distributed uniformly over a small ROI, since the ROI analysis at this spatial scale benefits from substantial signal averaging (Voormolen et al., 2010). Accordingly, both methods can provide different types of information and are thus considered as complementary (Giuliani et al., 2005).

While no VBM study to date has examined the effects of methylphenidate on GM volumes in ADHD patients, two metaanalyses investigated potential effects of medication indirectly through meta-regression analyses, based on variations in percentage of patients receiving medication included in published VBM studies of ADHD. These studies reported a 'normalizing' effect of psychostimulants in the caudate nucleus, the ACC and the amygdala (Nakao et al., 2011; Frodl and Skokauskas, 2012). Such meta-regression analyses are powerful tools, but due to their indirect nature, they are also characterized by intrinsic limitations. Here, for example, medication was assessed through the percentage of patients receiving medication in past studies, which does not take into account between-study variations in treatment duration, daily dosage, comorbidity rates, gender ratios, pre-processing options and/or statistical thresholding. Such variations in the underlying studies introduce confounds to the meta-analysis. Considering the widespread prescription of methylphenidate, more direct approaches should be used to gather convergent information and improve our understanding of methylphenidate's effects on brain development.

The present SMRI study is the first to use VBM to directly investigate GM volume differences in never-medicated children with $A D H D$, medicated children with $A D H D$ and TD children. VBM was used as a first step in an exploratory manner to investigate GM volume differences between groups throughout the whole brain. As a second step, to improve detection of potential diffuse differences in relevant structures of the brain, measurements of three selected ROls were obtained through automated tracing. Only structures of moderate size were selected (Voormolen et al., 2010), based on previous findings (Nakao et al., 2011, Frodl and Skokauskas, 2012, Hoekzema et al., 2014) and pharmacological plausibility (Volkow, 2012). These ROls were: the caudate nucleus, the amygdala and the nucleus accumbens. We hypothesized that medicated children with ADHD would exhibit less pronounced grey matter volume reductions than medication-naïve children with $A D H D$ in the caudate nucleus and in the amygdala compared to TD children, that no significant between-group difference would be found in the nucleus accumbens, and that there would be a significant correlation between treatment duration and GM volumes in the caudate nucleus and in the amygdala in medicated children with ADHD.

\section{Experimental procedures}

\subsection{Participants}

Eighty-four children (44 males) aged 7.3-12.9 participated in this study. Children with ADHD were recruited from the outpatient clinic in Erasme Hospital, Université libre de Bruxelles, Belgium. TD participants were recruited from local schools in Brussels or via personal request to professionals working at Erasme Hospital. Out of the 84 participants, six children with ADHD were excluded from the analysis following the discovery of anatomical brain abnormalities, and one child with ADHD was excluded due to excessive motion during MRI scanning. Analyses were thus conducted on 77 right-handed children (mean age $=10.2$ years; Standard deviation $(S D)=1.3$ years), comprising 53 children fulfilling DSM-IV-R criteria for the ADHD combined type and 24 TD children. The study groups were: TD children ( $n=24,12$ males), subjects with $A D H D$ with a history of long-term treatment with stimulants ( $n=20,16$ males), participants with ADHD who had no history of psychotropic medication treatment ( $n=33,18$ males). The three groups were comparable on age, gender, IQ estimate measured by the age-appropriate Wechsler Abbreviated Scale of Intelligence (WASI; Wechsler, 1999) and socio-economic status (SES) (Table 2). IQ estimates for medicated children with ADHD were obtained off-medication ( $24 \mathrm{~h}$ prior). Parental SES was assigned to one of three categories (unskilled/ qualified worker, clerk/ commercial occupation, and graduate occupation) by considering the profession of the most qualified parent. Diagnosis for ADHD was based on clinical features including typical history and behavioural report. The Kiddie Schedule for Affective Disorders and Schizophrenia for School Aged Children-Present and Lifetime Version (K-SADS-PL; Endicott and Spitzer, 1978) was completed at screening for each participant to establish the diagnosis according to DSM-IV-R criteria in children with ADHD, and to ensure that TD children presented no psychiatric condition. Symptoms' severity in children with $A D H D$ was measured using the ADHD rating scale parent form (parents of medicated children were asked to rate the behaviour of their child when off-medication) (DuPaul et al., 1998). Medicated children with $A D H D$ received between 0.3 and $0.5 \mathrm{mg}$ of methylphenidate per kilo (per dose, three times a day) for a minimum of one year (medication duration: more than one year, 11 children; more than 2 years, 7 children; between 3 and 4 years, 2 children) and no other medication. All other participants did not receive any medication.

Exclusion criteria for children with ADHD and TD children were presence of a psychiatric condition other than ADHD (as assessed by the K-SADS-PL), history of prematurity, current or past medical or neurological disorder, contraindication to MRI, and IQ estimate under 85. The TD children were naïve for any medication at the time of scanning. All subjects lived with their family, and were attending normal primary schools. Each child and her/his parents gave their written consent to participate in this study approved by the Ethics Committee of the Erasme University Hospital (reference: P2007/332/B40620072950).

\subsection{Image acquisition}

Participants were scanned using a 3 T Philips Achieva MRI scanner (Philips Healthcare, Best, The Netherlands) with an 8 channel SENSE head coil. A high-resolution, 3D T1-weighted structural scan was acquired using a sagittal TFE sequence with the following parameters: 160 slices; $T R=1960 \mathrm{~ms} ; \mathrm{TE}=4.60 \mathrm{~ms} ; \mathrm{TI}=1040 \mathrm{~ms}$; flip angle $=8^{\circ}$; field of view $=250 \mathrm{~mm} \times 250 \mathrm{~mm}$; matrix size $=320 \times 320$; reconstruction interpolated voxel size $=0.87 \times 0.87 \times 1.0 \mathrm{~mm}^{3}$.

\subsection{Behavioural data analyses}

Continuous demographic data were analysed using independent-sample $t$-tests and univariate analysis of variance (ANOVA). Categorical 
demographic data were analysed using Chi-Square tests. All analyses were carried out using the Statistical Package for Social Sciences version 17.0 (SPSS Inc., Chicago IL, USA).

\subsection{Voxel-based morphometry analysis}

Data were processed using the Statistical Parametric Mapping Software version 8 (SPM8; /http://www.fil.ion.ucl.ac.uk/spm/soft ware/spm8); Wellcome Department of Imaging Neuroscience, Lon don, UK) and the VBM8 Toolbox. Customised tissue probability maps were produced using the matched template approach of the Template-O-Matic Toolbox for SPM8, with each participant's age and gender as defining variables. First, all T1-weighted images were checked for scanner- and individual-based artefacts. Individual images were then corrected for bias-field inhomogeneities, segmen ted and spatially normalised (affine-only transformation) with reference to customised tissue probability maps. Segmentation accuracy was visually checked for each participant. Based on individual registered grey matter (GM) and white matter (WM) segmentations, an average DARTEL (Diffeomorphic Anatomical Registration Through Exponentiated Lie Algebra) template of all participants was created in the MNI space (Ashburner and Friston, 2000). The affine-registered GM segments were then warped to this average template using the high-dimensional DARTEL approach and modulated. Crucially, the voxel's signal intensity values in the grey matter segments were only multiplied by the non-linear component of the registration to account for individual differences in brain size. Finally, the GM segments were smoothed using a $5 \times 5 \times 5 \mathrm{~mm}^{3}$ full-width-at-half maximal Gaussian kernel (Ashburner and Friston, 2000).

Statistical analyses were subsequently performed on a voxel-byvoxel basis employing the framework of the general linear model (GLM) within SPM8, with a three-group comparison design contrasting the medicated $A D H D$, never-medicated $A D H D$ groups versus the TD group. Using an absolute threshold of 0.1 , regionally-specific between-group differences in grey matter volume were assessed, with gender and age as covariates of no interest. Results were examined at the whole brain level, and considered significant at the statistical threshold of $p<0.001$ uncorrected, using an empirically determined extent threshold of 38 contiguous voxels to protect against type 1 error (expected number of voxels according to random field theory, i.e. the expected Euler characteristic of the image was used to calculate a threshold for the required control of false positives) (Nenadic et al., 2010). A mean grey matter volume estimate for each significant cluster was extracted using the Marsbar Toolbox. When significant differences involving medicated children with ADHD were found, correlations between duration of medication and mean grey matter volume estimate within the significant cluster (age and gender regressed out) were examined.

\subsection{ROI analysis}

Total intracranial volume and ROI volumes were estimated using the standard automated cortical and subcortical segmentations in Freesurfer (〈http://surfer.nmr.mgh.harvard.edu〉). Summary images for the outputs were generated and inspected to check segmenta tion quality. We extracted volumes of our 6 a priori ROls (left and right caudate nucleus, amygdala and nucleus accumbens) in each of the 77 participants. These volume measurements were entered into a GLM (3 groups comparison ANOVA, total intracranial volume regressed out), with gender and age as covariates of no interest. Since our design included 3 groups, Fisher's LSD post-hoc tests were conducted following significant $F$-test. In addition, we examined correlations between the volume of each region and duration of medication exposure. Because we examined six different $a$ priori regions, we performed a bonferroni correction on the $p$-values resulting from the post-hoc tests and correlation analyses $(p=0.05$ / $6=0.0083$ ).

\section{Results}

\subsection{Demographic characteristics}

Analyses revealed no significant differences between the medicated ADHD, never-medicated ADHD and TD groups for gender, IQ, age or SES (Table 1). Medicated and nevermedicated children with ADHD did not differ on ratings of ADHD symptoms' severity (Table 1).

Table 1 Demographic characteristics and brain volumes of participants: never-medicated ADHD vs. Medicated ADHD vs. TD.

\begin{tabular}{|c|c|c|c|c|c|c|c|}
\hline \multirow[t]{2}{*}{ Measure } & \multicolumn{6}{|l|}{ Group } & \multirow[t]{2}{*}{ Analysis } \\
\hline & \multicolumn{2}{|l|}{$\begin{array}{l}(1) \mathrm{TD} \\
(n=24)\end{array}$} & \multicolumn{2}{|c|}{$\begin{array}{l}\text { (2) Medicated ADHD } \\
(n=20)\end{array}$} & \multicolumn{2}{|c|}{$\begin{array}{l}\text { (3) Never-medicated ADHD } \\
(n=33)\end{array}$} & \\
\hline \multirow[t]{2}{*}{ \%Male } & $50 \%$ & & $80 \%$ & & $55 \%$ & & $.09^{\mathrm{a}}$ \\
\hline & Mean & SD & Mean & SD & Mean & SD & $P$-value \\
\hline Age, years & 10 & 1.2 & 10.4 & 1.4 & 10.3 & 1.4 & .47 \\
\hline IQ & $109.7^{\mathrm{b}}$ & 8.6 & $107.4^{c}$ & 13.5 & $105.6^{c}$ & 10.7 & .43 \\
\hline SES & 2.6 & 0.5 & 2.5 & 0.7 & 2.3 & 0.8 & .14 \\
\hline ADHD Scores ${ }^{d}$ & & & 39.6 & 6.8 & 38 & 5.4 & .36 \\
\hline
\end{tabular}

Abbreviations: ADHD, Attention-deficit/Hyperactivity Disorder; CSF, Cerebro-spinal fluid; GM, Grey matter; IQ, Intelligence quotient; SD, Standard deviation; SES, Socio-economic status; TD, Typically developing

a Pearson $\chi^{2}$ test. Age, IQ and SES were analyzed using Student $t$-tests, and total volumes were analyzed within an ANOVA with age and sex as covariates of no interest.

${ }^{\mathrm{b}}$ Two data missing.

${ }^{\mathrm{C}}$ One data missing for each group.

${ }^{\mathrm{d}}$ ADHD Rating Scale-IV home version, parent's ratings. 


\subsection{Voxel-based morphometry}

ADHD never-medicated versus TD children: when compared with TD children, never-medicated children with ADHD displayed decreased GM volume in the insula and in the middle temporal lobe (Table 2).

ADHD medicated versus TD children: Medicated children with $A D H D$ exhibited increased $G M$ volume in the middle frontal gyrus and in the precentral gyrus when compared with TD children (Table 2). In medicated children, there was no significant correlation between treatment duration and volumes in the middle frontal gyrus $(r=-.17 ; p=.45)$ or in the precentral gyrus $(r=-.01 ; p=.95)$.

$A D H D$ medicated versus never-medicated children: When compared to the medicated children, the never-medicated children displayed increased GM volume in the middle frontal gyrus, and reduced GM volume in the putamen, the insula, the posterior lobe of the cerebellum and the

Table 2 Voxel-based morphometry analyses. Whole brain group differences, $p<.001$ uncorrected, with an empirically determined extent threshold of 32 voxels.

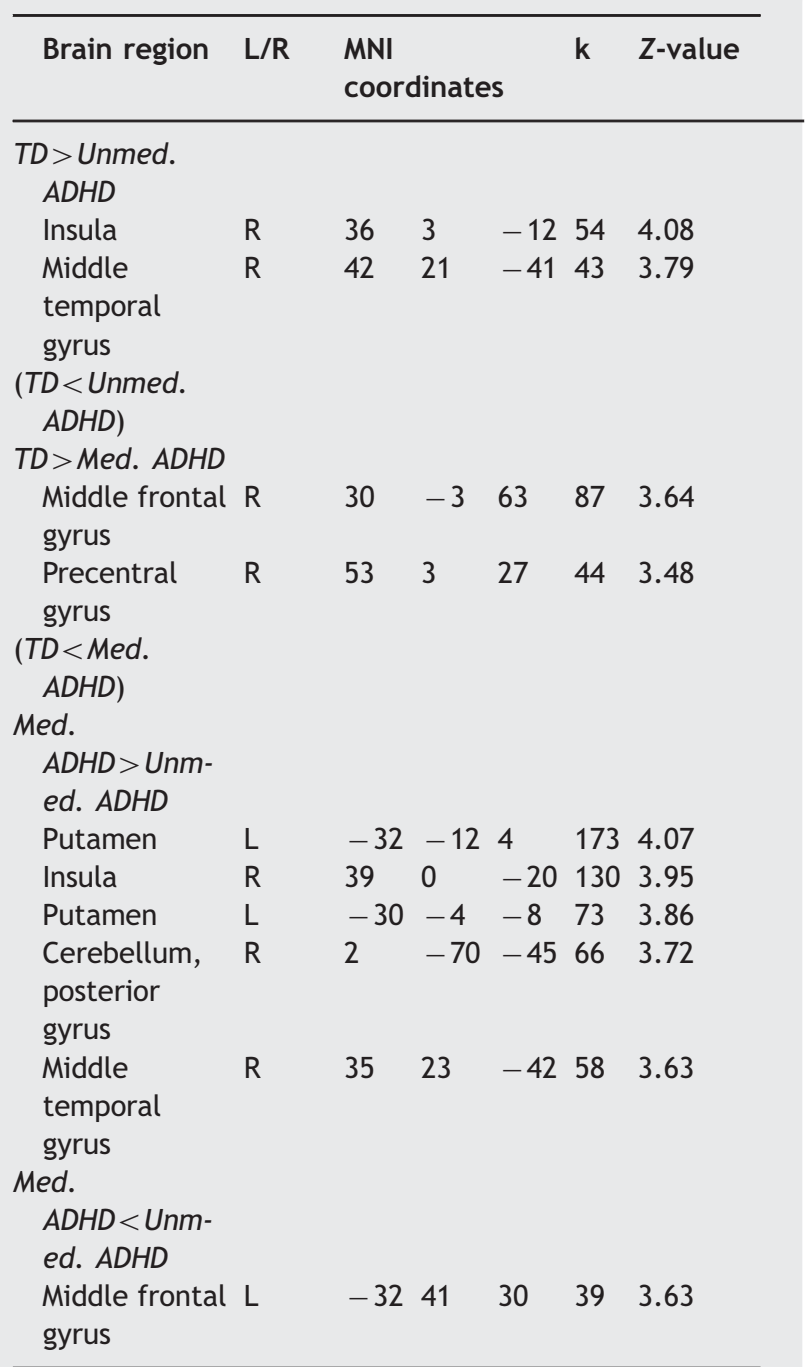

Abbreviations: L/R - left/right; MNI - Montreal Neurological Institute; $k$ - cluster size; Med., Medicated; Unmed., Unmedicated; TD, Typically Developing. middle temporal gyrus (Table 2). In medicated children, there was no significant correlation between treatment duration and volumes in the middle frontal gyrus $(r=-.28$; $p=.38$ ), the putamen (first coordinate: $r=.12 ; p=.63$; second coordinate: $r=-.15 ; p=.55)$, the insula $(r=.05$; $p=.81)$, the cerebellum $(r=-.14 ; p=.54)$ or the middle temporal gyrus $(r=-.20 ; p=.39)$.

\subsection{ROI measurements}

We found no significant difference between our three groups in any of the 6 a priori regions examined (Table 3). In medicated children with ADHD, correlational analyses revealed a positive and significant correlation between treatment duration and GM volume of the left nucleus accumbens area ( $r=.59 ; p=.008$; Table 3; Figure 1 and 2$)$.

\section{Discussion}

This sMRI study is the first to use VBM to directly compare GM volumes in medicated and never-medicated children with ADHD, and TD children. When compared to both medicated children with $\mathrm{ADHD}$ and TD children, nevermedicated children with ADHD presented decreased GM volume in the insula and in the middle temporal gyrus. When compared to TD children, medicated children with ADHD exhibited decreased GM volume in the middle frontal gyrus and in the precentral gyrus. Differences in GM volume between medicated and never-medicated children with ADHD were also found in the putamen, cerebellum (Medicated $>$ Never-Medicated) and middle frontal gyrus (Medicated $<$ Never-Medicated). Finally, ROI analyses revealed a significant association between duration of treatment and GM volume of the left nucleus accumbens in medicated children with ADHD.

In this study, GM volume reductions were found in nevermedicated children with ADHD in the insula and in the middle temporal gyrus when compared both with medicated children with ADHD and TD children. In past sMRI studies in $A D H D$ relying on ROls measurements, such a pattern of results was usually interpreted as suggesting a 'normalizing' effect of methylphenidate on GM volumes (SemrudClikeman et al., 2006; Bledsoe et al., 2009). This interpretation is questionable, for at least two reasons. First, ROI analysis relies solely on a single average measure for the structure of interest. This averaging may offer an overly simplistic view of complex local dynamics involving both increases and decreases in GM volume within the structure. Second, cross-sectional designs cannot control for nontreatment-related differences between medicated and never-medicated groups. Over the years, ADHD has proven to be an extremely heterogeneous disorder, involving multiple neural pathways (Villemonteix et al., 2014), which probably explains why findings from individual studies examining disorder-related structural deficits have been so inconsistent (Nakao et al., 2011). Therefore, nevermedicated and medicated groups recruited in past crosssectional studies might present on average with different neuropsychological and neurobiological characteristics, which is a confounding factor when examining treatment effects. 
Table 3 ROI measures of the caudate nucleus, the amygdala and the nucleus accumbens.

\begin{tabular}{|c|c|c|c|c|c|c|c|c|c|c|}
\hline \multirow[t]{2}{*}{ Brain region } & \multicolumn{2}{|l|}{ TD } & \multicolumn{2}{|c|}{ Med. ADHD } & \multicolumn{2}{|c|}{ Unmed. ADHD } & \multicolumn{2}{|c|}{ GLM results } & \multicolumn{2}{|c|}{ Correlation results } \\
\hline & Mean & SD & Mean & SD & Mean & SD & $F$ test & Adjusted $p$ & $r$ & $p$ \\
\hline Caudate L & 3850.8 & 521.6 & 4062.9 & 718.1 & 4162.4 & 570.5 & 1.4 & .25 & .23 & .334 \\
\hline Caudate R & 3920.2 & 495.9 & 4135.7 & 663.2 & 4193.3 & 601.2 & 1.0 & .37 & .23 & .344 \\
\hline Amygdala L & 1725.4 & 229.4 & 1795.7 & 250.5 & 1754.4 & 271.9 & 0.7 & .93 & .38 & .105 \\
\hline Amygdala R & 1806.5 & 226.6 & 1967.7 & 257.6 & 1937.4 & 268.3 & 2.0 & .14 & .28 & .237 \\
\hline Accumbens L & 716.7 & 152 & 800.7 & 124.7 & 773.6 & 112.6 & 2.2 & .11 & .59 & .008 \\
\hline Accumbens $\mathrm{R}$ & 791.5 & 112.7 & 872.6 & 150.1 & 845.6 & 122.3 & 1.88 & .16 & .30 & .206 \\
\hline
\end{tabular}

Abbreviations: GLM, General Linear Model; L/R - left/right; MNI - Montreal Neurological Institute; k - cluster size; Med., Medicated; Unmed., Unmedicated: TD, Typically Developing. Volume is indicated in cubic millimeters. For GLM results, group differences between the 3 groups are reported as statistics. Individual subject values were covaried in a GLM to account for age and sex, yielding $F$ and adjusted $p$-values. For Correlation results, correlations between GM volume and medication duration in medicated children with ADHD $(n=20)$ are reported. $P$-values significant after applying a Bonferroni correction are shown in bold.
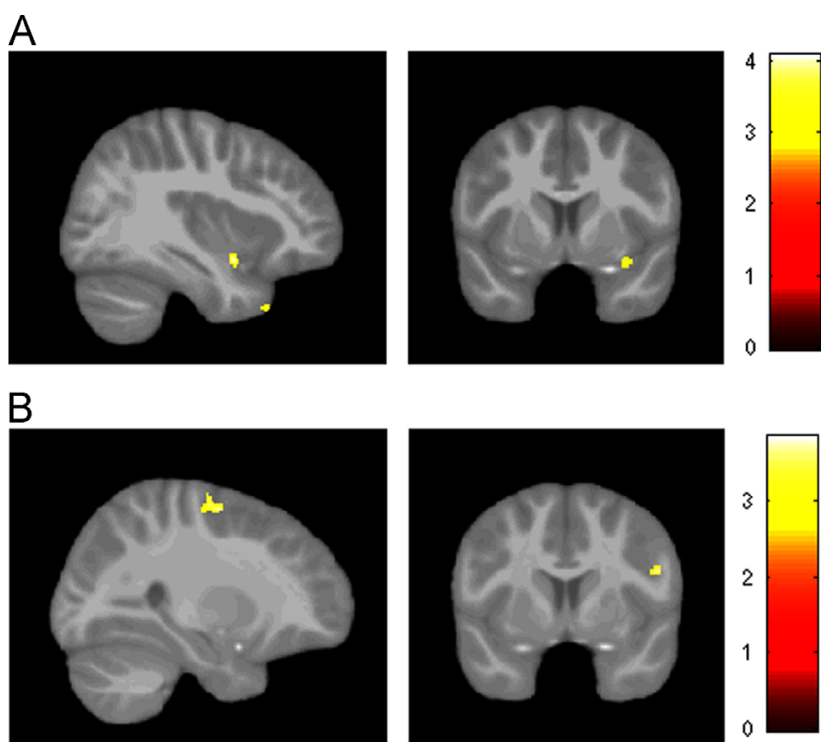

Figure 1 Statistical Parametric Maps (SPMs) showing foci of significant reduced grey matter volume (sagittal and coronal views) among $A$ ) the never-medicated children with ADHD $(n=33)$ relative to the TD children $(n=24)$ in the insula and in the middle temporal gyrus, and $B$ ) the medicated children $(n=20)$ with ADHD relative to the TD children $(n=24)$ in the middle frontal gyrus and precentral gyrus.

Here, VBM allowed us to investigate GM volume differences within brain structures, at the cluster level, which strengthens the interpretation of findings. Nevertheless, similarly to all previous SMRI studies of treatment effects on GM volumes in ADHD but two (Castellanos et al., 2002; Shaw et al., 2014), our design was both naturalistic and cross-sectional. For this reason, while our medicated and never-medicated groups both exhibited non-comorbid combined-type ADHD and did not differ in levels of symptom's severity, they may still be characterized on average by different disorder-related structural abnormalities. In this context, the relationship between group-comparison findings and treatment effects should be discussed carefully, based on the convergence of different levels of analysis.

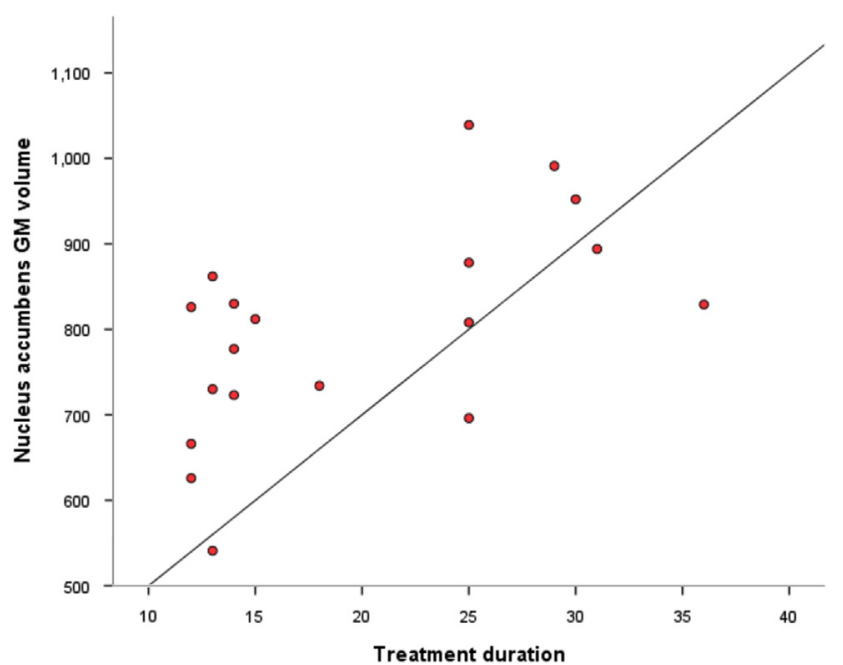

Figure 2 Medicated children with ADHD. Scatter plots showing the positive correlation between GM volume of the nucleus accumbens (in cubic millimeters) and duration of exposure to methylphenidate (in months). Abbreviations: GM, Grey matter.

The insula is known to be involved in the assessment of motivational and rewarding aspects of tasks, as well as in monitoring of task difficulty and performance (Ivanov et al., 2014). Interestingly, meta-analyses of functional MRI (fMRI) studies reported an altered functioning in this region in patients with ADHD during working memory, inhibition and attention tasks (Cortese et al., 2010), and found that psychostimulants most consistently increase insula and right inferior frontal gyrus activations during stop and time discrimination tasks (Rubia et al., 2014). Also, increased grey matter volumes in the insula have recently been reported in young occasional users of amphetamine-type psychostimulants such as methylphenidate (Mackey et al., 2014). Based on these results, our finding of increased grey matter volumes in medicated children with ADHD in the insula when compared with never-medicated children with ADHD may be suggestive of a structural normalization following chronic methylphenidate administration. In this case, the inclusion of a majority of medicated participants may explain why previous VBM studies of ADHD did not 
report grey matter volume reductions in this brain region in the patient groups (Nakao et al., 2011).

ADHD related-middle temporal lobe abnormalities have been reported in the past in one fMRI study during a divided attention task (Shafritz et al., 2004), but not consistently in meta-analyses (Cortese et al., 2010). Also, methylphenidate administration had no direct effect on ADHD-related hypoactivations in this brain region (Shafritz et al., 2004). Here, never-medicated children with ADHD presented decreased GM volume in the anterior end of the middle temporal lobe (Brodmann area 38, temporal pole) when compared to medicated children with ADHD and TD children. Reduced cortical thickness in the anterior temporal lobe has been reported in the past in adults with childhood ADHD at 33-year follow-up when compared with controls, with more pronounced cortical thinning in this region in adults with persistent ADHD when compared with the nonADHD group (Proal et al., 2011). Interestingly, in the only previous SMRI study investigating treatment effects at the whole brain level, the anterior temporal lobe was the only region showing significant differences in cortical thickness between medicated and never-medicated children with ADHD (Shaw et al., 2006). Long considered as enigmatic, this part of the temporal lobe is thought to be a cortical convergence zone, binding highly processed perceptual inputs to visceral emotional responses (Proal et al., 2011). Structural abnormalities in the temporal pole may therefore partly underlie the abnormalities in emotional processing found in patients with ADHD (Villemonteix et al., 2014), and methylphenidate exposure may contribute to reduce disorder related-structural abnormalities in this brain region.

In the present study, decreased GM volumes were found in the middle frontal gyrus (brodmann area 6; premotor cortex) and in the precentral gyrus (brodmann area 9) in medicated children with ADHD when compared with TD children. The premotor cortex is known to be involved in the planning of complex coordinated movements, and decreased grey matter volumes have been found in the past in this brain region in children with ADHD (Mostofsky et al., 2002). The precentral gyrus (B.A. 9) is implicated in multiple executive functions, such as sustained attention, working memory and response inhibition (Suskauer et al., 2008). By blocking norepinephrine transporters, methylphenidate leads to increased extracellular catecholamine levels in frontal regions (Rubia et al., 2014). However, treatment-related changes in brain activation during cognitive tasks have usually been found in inferior frontal regions, as opposed to these middle frontal locations (Rubia et al., 2014). Therefore, previous literature provides no solid ground to relate these GM volume changes to treatment effects.

When considering these findings, one must note that no significant correlation between treatment duration and GM volumes were found in these four brain regions in medicated children with ADHD. Due to this lack of convergence between correlational analyses and between-group comparisons, we cannot firmly conclude that the significant between-group differences represent treatment effects, all the more so as our study is the first to directly investigate GM volume differences associated with exposure to methylphenidate in these brain regions. Findings should therefore be considered as preliminary until replicated in other VBM studies of medication's effects in ADHD.
In this study, ROI analyses were also used to improve detection of more diffuse structural alterations within brain structures of moderate size (Voormolen et al., 2010). This set of analyses yielded one significant finding: in medicated children with ADHD, GM volume of the left nucleus accumbens was positively associated with treatment duration. In the only previous study investigating methylphenidate effects on GM volume in the nucleus accumbens in children with ADHD, Hoekzema and colleagues reported an initial bilateral reduction in volume in this region, followed by a recovery of volume (Hoekzema et al., 2014). However, children participating in this study were all medicated for a maximum of 13 months (mean duration: 7.5 months). In our study, treatment durations all exceeded one year. Therefore, our findings suggest that longer treatment durations may be associated with increased GM volume in the nucleus accumbens, at least in the left part. This would be in line with previous studies reporting increases of the left nucleus accumbens volume following exposure to amphetamines, especially in young users (Jan et al., 2012). In rats, exposure to methylphenidate has also been shown to increase the density of dendritic spines of medium-sized spiny neurons in the nucleus accumbens (Kim et al., 2009). Finally, in the three-group comparison analysis, there was a non-significant trend towards increased GM volume in the left nucleus accumbens in medicated children with ADHD when compared to both never-medicated children with ADHD and TD children (Table 3). One can wonder whether longer treatment duration may yield a significant result for this contrast.

Considering the key role played by the nucleus accumbens in addiction (Volkow, 2012), methylphenidate-related potential structural changes in this region should be discussed in relation with substance abuse liability (Robinson and Kolb, 1997, 2004; Russo et al., 2010). Studies in rodents have shown that methylphenidate self-administration enhances the potency and reinforcing efficacy of amphetamine-related drugs in the nucleus accumbens (Calipari and Jones, 2014), suggesting that methylphenidate may change the dopamine system in a way that promotes future drug abuse. Nonetheless, development of sensitization and related behaviors depends on the nature of the treatment regimen, and it is not clear to what extend drug exposure paradigms in rodents mirror the long-term methylphenidate usage in children with ADHD (Kuczenski and Segal, 2005). Studies in non-human primates reported no significant association between chronic treatment with methylphenidate and vulnerability for cocaine addiction, and no medication-related effect on dopamine systems development (Volkow, 2012). In humans, most studies seem to find no or possibly protective effects of ADHD medication on substance abuse (Humphreys et al., 2013; Chang et al., 2014). However, two studies found an association between age of initiation of methylphenidate treatment and substance abuse, such as subjects with late initiation of stimulant medication (ages 8-12) displayed greater substance use (Mannuzza et al., 2008; Dalsgaard et al., 2014). In one of these studies, the association was found to be mediated by increasing rates of antisocial personality disorder diagnosis (Mannuzza et al., 2008).

Interestingly, increased grey matter volumes in the left nucleus accumbens have been reported in individuals displaying antisocial tendencies (Schiffer et al., 2011). Also, 
while individuals displaying antisocial tendencies have long been thought to exhibit abnormalities in reward processing, impulsive-antisocial traits have been found to predict dopamine release and reward anticipation-related neural activity in the nucleus accumbens in response to pharmacological and monetary reinforcers (Buckholtz et al., 2010). Building on these lines of evidence, one can wonder whether methylphenidate related-structural modifications in the left nucleus accumbens may subtend abnormalities in reward processing in at least a subset of medicated children with ADHD. In line with this hypothesis, animal studies have reported abnormalities in reward processing in rodents following exposure to methylphenidate (Bolanos et al., 2003, Warren et al., 2011). However, another study in rodent suggested that structural alterations of the nucleus accumbens following methylphenidate exposure may act as a protective factor against impulsive behaviour (Leo et al., 2009). More structural and functional brain imaging studies examining long term effects of chronic exposure to methylphenidate in the nucleus accumbens are needed to clarify this issue.

Contrary to our hypothesis, we found no GM volume difference between never-medicated children with ADHD and TD children in the caudate nucleus or in the amygdala, nor treatment effects in these regions. ADHD is a highly heterogeneous condition: reduced GM volume in children with $A D H D$ in the caudate nucleus are one of the most replicated findings in SMRI studies (Nakao et al., 2011; Frodl and Skokauskas, 2012), but structural deficits in this region are not expected to be found in all subgroups of children with ADHD. Similarly, previous studies have been inconsistent regarding treatment effects. Only one meta-analysis reported medication-related structural changes in the amygdala (Frodl and Skokauskas, 2012), and a recent longitudinal study reported no treatment effects in the caudate nucleus (Shaw et al., 2014).

Several methodological limitations should be noted. First, due to the exploratory nature of our study, we decided not to apply the most conservative statistical thresholding (i.e. Family Wise Error Rate correction for multiple comparisons) to our VBM results. Instead, results were considered significant at $p<0.001$ uncorrected, using an empirically determined extent threshold (Nenadic et al., 2010). Second, in medicated children, duration of exposure to methylphenidate did not exceed four years. Longer exposure may be required to detect significant impact on brain structures. Third, GM volumes are only one among many indicators of brain development. Future studies should consider medication's effects over longer periods, using longitudinal designs including detailed clinical assessments in conjunctions with other indices of structural brain connectivity. This study was also characterized by several strengths. First, the use of two complementary approaches (VBM and ROI measurements) allowed us to optimally investigate both diffuse and focal structural differences between groups (Voormolen et al., 2010). Second, our study included the second largest ADHD sample to date when compared with previous VBM studies. Third, one must note that all our children with ADHD had no comorbid psychiatric disorder. While this means that our sample was somewhat different from typical samples of referred children with $A D H D$ showing high rates of comorbidity, it also means that children included in our study presented a 'purest' form of the disorder, possibly leading to a more homogeneous sample than those included in previous VBM studies.

In conclusion, in this cross-sectional study of methylphenidate's effects on GM volumes in ADHD, we report evidence of potential treatment-related GM volume normalization in the insula and in the middle temporal gyrus, as well as potential neurodevelopmental deviations in the middle frontal and precentral gyri. However, due to the crosssectional nature of our design, these findings should be considered as preliminary until replicated. We also document a significant association between medication history and GM volume in the left nucleus accumbens, a region known for its key role in reward-processing and addictions. Structural and functional effects of methylphenidate in this brain structure undoubtedly deserve further investigation. Despite a decade of research, knowledge about methylphenidate's long term effects remain scarce. More structural studies using both cross-sectional and longitudinal are needed to gather convergent information and inform public debates on this sensitive issue.

\section{Role of the funding source}

This work was supported by a grant from the Belgian National Fund for Scientific Research (FNRS 3.4.516.08.F). Stéphane A. De Brito was supported by a research fellowship from the Swiss National Science Foundation (SNSF PAOOP1_139586). The funding sources had no involvement in study design, in the collection, analysis or interpretation of data, in the writing of the report and in the decision to submit the paper for publication.

\section{Contributors}

Thomas Villemonteix: analysis, interpretation of data, drafted the first version of the article; Stéphane A. De Brito: Analysis, interpretation of data; Hichem Slama: acquired data, interpretation of data; Martin Kavec: technical conception, acquired data; Danielle Balériaux: technical conception, acquired data; Thierry Metens: technical conception, acquired data. Simon Baijot: acquired data, interpretation of data; Alison Mary: acquired data, interpretation of data; Philippe Peigneux: principal investigator, conception, design, interpretation of data; Isabelle Massat: principal investigator, conception, design, interpretation of data. All authors contributed to and have approved the final manuscript.

\section{Conflict of interest}

Isabelle Massat received compensations as a speaker in local educational meeting devoted to ADHD in 2013 and financial aid for registration for the ADHD World Congress in 2014 from Shire Company. Thomas Villemonteix received financial aid for travel expenses during the ADHD World Congress in 2014 from Shire Company. All other authors report no conflict of interest.

\section{Acknowledgments}

This work was supported by a grant from the Belgian National Fund for Scientific Research (FNRS 3.4.516.08.F). Stéphane A. De Brito was supported by a research fellowship from the Swiss National Science Foundation (SNSF PA00P1_139586). The authors thank all children 
and their families for their participation, and Mustapha Nouali and his team for kind help and assistance in MRI data collection.

\section{References}

Ashburner, J., Friston, K.J., 2000. Voxel-based morphometry - the methods. Neurolmage 11, 805-821.

Biederman, J., Faraone, S.V., 2005. Attention-deficit hyperactivity disorder. Lancet 366, 237-248.

Bledsoe, J., Semrud-Clikeman, M., Pliszka, S.R., 2009. A magnetic resonance imaging study of the cerebellar vermis in chronically treated and treatment-naïve children with attention-deficit/ hyperactivity disorder combined type. Biol. Psychiatry 65, 620-624.

Bolanos, C.A., Barrot, M., Berton, O., Wallace-Black, D., Nestler, E. J., 2003. Methylphenidate treatment during pre- and periadolescence alters behavioral responses to emotional stimuli at adulthood. Biol. Psychiatry 54, 1317-1329.

Buckholtz, J.W., Treadway, M.T., Cowan, R.L., Woodward, N.D., Benning, S.D., Li, R., Ansari, M.S., Baldwin, R.M., Schwartzman, A.N., Shelby, E.S., Smith, C.E., Cole, D., Kessler, R.M., Zald, D. H., 2010w. Mesolimbic dopamine reward system hypersensitivity in individuals with psychopathic traits. Nat. Neurosci. 13, 419-421.

Calipari, E.S., Jones, S.R., 2014. Sensitized nucleus accumbens dopamine terminal responses to methylphenidate and dopamine transporter releasers after intermittent-access self-administration. Neuropharmacology 82, 1-10.

Castellanos, F.X., Lee, P.P., Sharp, W., Jeffries, N.O., Greenstein, D. K., Clasen, L.S., Blumenthal, J.D., James, R.S., Ebens, C.L., Walter, J.M., Zijdenbos, A., Evans, A.C., Giedd, J.N., Rapoport, J.L., 2002. Developmental trajectories of brain volume abnormalities in children and adolescents with attention-deficit/hyperactivity disorder. JAMA-J. Am. Med. Assoc. 288, 1740-1748.

Chang, Z., Lichtenstein, P., Halldner, L., D’Onofrio, B., Serlachius, E., Fazel, S., Langstrom, N., Larsson, H., 2014. Stimulant ADHD medication and risk for substance abuse. J. Child Psychol. Psychiatry 55, 878-885.

Cortese, S., Kelly, C., Chabernaud, C., Proal, E., Di Martino, A., Milham, M.P., Castellanos, X., 2010w. Towards systems neuroscience of ADHD: a meta-analysis of 55 fMRI studies. Am. J. Psychiatry 169, 1038-1055.

Dalsgaard, S., Mortensen, P.B., Frydenberg, M., Thomsen, P.H., 2014. ADHD, stimulant treatment in childhood and subsequent substance abuse in adulthood - a naturalistic long-term followup study. Addict. Behav. 39, 325-328.

DuPaul, G.J., Power, T.J., Anastopoulos, AD, Reid, R, 1998. ADHD Rating Scale-IV: Checklists, Norms, and Clinical Interpretation ed. The Guilford Press, New York.

Endicott, J., Spitzer, R.L., 1978. A diagnostic interview. The schedule for affective disorders and schizophrenia. Arch. Gen. Psychiatry 35, 837-844.

Frodl, T., Skokauskas, N., 2012. Meta-analysis of structural MRI studies in children and adults with attention deficit hyperactivity disorder indicates treatment effects. Acta Psychiatr. Scand. $125,114-126$.

Giuliani, N.R., Calhoun, V.D., Pearlson, G.D., Francis, A., Buchanan, R.W., 2005w. Voxel-based morphometry versus region of interest: a comparison of two methods for analyzing gray matter differences in schizophrenia. Schizophr. Res. 74, 135-147.

Humphreys, K.L., Eng, T., Lee, S.S., 2013w. Stimulant medication and substance use outcomes a meta-analysis. JAMA Psychiatry 70, 740-749.

Hoekzema, E., Carmona, S., Ramos-Quiroga, J.A., Canals, C., Moreno, A., RicharteFernandez, V., Picado, M., Bosch, R., Duno, L., Soliva, J.C., Rovira, M., Bulbena, A., Tobena, A., Casas, M., Vilarroya, O., 2014. Stimulant drugs trigger transient volumetric changes in the human ventral striatum. Brain Struct. Func. 219, 23-34.

Ivanov, I., Bansal, R., Hao, X., Zhu, H., Kellendonk, C., Miller, L., Sanchez-Pena, J., Miller, A.M., Chakravarty, M.M., Klahr, K., Durkin, K., Greenhill, L.L., Peterson, B.S., 2010. Morphological abnormalities of the thalamus in youths with attention deficit hyperactivity disorder. Am. J. Psychiatry 167, 397-408.

Ivanov, I., Liu, X., Clerkin, S., Schulz, K., Fan, J., Friston, K., London, E.D., Schwartz, J., Newcorn, J.H., 2014. Methlyphenidate and brain activity in a reward/conflict paradigm: role of the insula in task performance. Eur. Neuropsychopharmacol.

Jan, R.K., Lin, J.C., Miles, S.W., Kydd, R.R., Russell, B.R., 2012. Striatal volume increases in active methamphetamine-dependent individuals and correlation with cognitive performance. Brain Sci. 2, 553-572.

Kim, Y., Teylan, M.A., Baron, M., Sands, A., Nairn, A.C., Greengard, P., 2009. Methylphenidate-induced dendritic spine formation and Delta FosB expression in nucleus accumbens. Proc. Natl. Acad. Sci. USA 106, 2915-2920.

Kroutil, L.A., Van Brunt, D.L., Herman-Stahl, M.A., Heller, D.C., Bray, R.M., Penne, M.A., 2006. Nonmedical use of prescription stimulants in the United States. Drug Alcohol Depend. 84, 135-143.

Kuczenski, R., Segal, D.S., 2005w. Stimulant actions in rodents: implications for attention-deficit/hyperactivity disorder treatment and potential substance abuse. Biol. Psychiatry 57, 1391-1396.

Leo, D., Adriani, W., Cavaliere, C., Cirillo, G., Marco, E.M., Romano, E., Di Porzio, U., Papa, M., Perrone-Capano, C., Laviola, G., 2009. Methylphenidate to adolescent rats drives enduring changes of accumbal Htr7 expression: implications for impulsive behavior and neuronal morphology. Genes, Brain Behav. 8, 356-368.

Levy, S., Katusic, S.K., Colligan, R.C., Weaver, A.L., Killian, J.M., Voigt, R.G., Barbaresi, W.J., 2014. Childhood ADHD and risk for substance dependence in adulthood: a longitudinal, populationbased study. Plos One, 9.

Mackey, S., Stewart, J.L., Connolly, C.G., Tapert, S.F., Paulus, M.P., 2014. A voxel-based morphometry study of young occasional users of amphetamine-type stimulants and cocaine. Drug Alcohol Depend. 135, 104-111.

Mannuzza, S., Klein, R.G., Truong, N.L., Moulton III, J.L., Roizen, E. R., Howell, K.H., 2008. Age of methylphenidate treatment initiation in children with $A D H D$ and later substance abuse: prospective follow-up into adulthood. Am. J. Psychiatry 165 , 604-609.

Mostofsky, S.H., Cooper, K.L., Kates, W.R., Denckla, M.B., Kaufmann, W.E., 2002w. Smaller prefrontal and premotor volumes in boys with attention-deficit/hyperactivity disorder. Biol. Psychiatry 52, 785-794.

Nakao, T., Radua, J., Rubia, K., Mataix-Cols, D., 2011w. Gray matter volume abnormalities in ADHD: Voxel-based meta-analysis exploring the effects of age and stimulant medication. Am. J. Psychiatry 168, 1154-1163.

Nenadic, I., Smesny, S., Schlösser, R.G., Sauer, H., Gaser, C., 2010. Auditory hallucinations and brain structure in schizophrenia: voxel-based morphometry study. Br. J. Psychiatry 196, 412-413.

Proal, E., Reiss, P.T., Klein, R.G., Mannuzza, S., Gotimer, K., RamosOlazagasti, M.A., Lerch, J.P., He, Y., Zijdenbos, A., Kelly, C., Milham, M.P., Castellanos, X., 2011. Brain gray matter deficits at 33-Year follow-up in adults with attention-deficit/hyperactivity disorder established in childhood. Arch. Gen. Psychiatry 68, 1122-1134.

Robinson, T.E., Kolb, B., 1997. Persistent structural modifications in nucleus accumbens and prefrontal cortex neurons produced by previous experience with amphetamine. J. Neurosci. 17, 8491-8497.

Robinson, T.E., Kolb, B., 2004. Structural plasticity associated with exposure to drugs of abuse. Neuropharmacology 47, 33-46. 
Rubia, K., Alegria, A.A., Cubillo, A.I., Smith, A.B., Brammer, M.J., Radua, J., 2014. Effects of stimulants on brain function in attention-deficit/hyperactivity disorder: a systematic review and meta-analysis. Biol. Psychiatry 76, 616-628.

Russo, S.J., Dietz, D.M., Dumitriu, D., Morrison, J.H., Malenka, R.C., Nestler, E.J., 2010. The addicted synapse: mechanisms of synaptic and structural plasticity in nucleus accumbens. Trends Neurosci. 33, 267-276.

Schiffer, B., Mueller, B.W., Scherbaum, N., Hodgins, S., Forsting, M., Wiltfang, J., Gizewski, E.R., Leygraf, N., 2011. Disentangling structural brain alterations associated with violent behavior from those associated with substance use disorders. Arch. Gen. Psychiatry 68, 1039-1049.

Schweren, L.J.S., de Zeeuw, P., Durston, S., 2013w. MR imaging of the effects of methylphenidate on brain structure and function in attention-deficit/hyperactivity disorder. Eur. Neuropsychopharmacol. 23, 1151-1164.

Semrud-Clikeman, M., Pliszka, S.R., Lancaster, J., Liotti, M., 2006. Volumetric MRI differences in treatment-naïve vs chronically treated children with ADHD. Neurology 67, 1023-1027.

Shafritz, K.M., Marchione, K.E., Gore, J.C., Shaywitz, S.E., Shaywitz, B.A., 2004. The effects of methylphenidate on neural systems of attention in attention deficit hyperactivity disorder. Am. J. Psychiatry 161, 1990-1997.

Shaw, P., Lerch, J., Greenstein, D., Sharp, W., Clasen, L., Evans, A., Giedd, J., Castellanos, F.X., Rapoport, J., 2006w. Longitudinal mapping of cortical thickness and clinical outcome in children and adolescents with attention-deficit/hyperactivity disorder. Arch. Gen. Psychiatry 63, 540-549.

Shaw, P., Sharp, W., Morrison, M., Eckstrand, K., Greenstein, D., Clasen, L., Evans, A., Rapoport, J., 2009. Psychostimulant treatment and the developing cortex in attention-deficit/hyperactivity disorder. Am. J. Psychiatry 166, 58-63.

Shaw, P., De Rossi, P., Watson, B., Wharton, A., Greenstein, D., Raznahan, A., Sharp, W., Lerch, J.P., Chakravarty, M.M., 2014. Mapping the development of the basal ganglia in children with attention-deficit/hyperactivity disorder. J. Am. Acad. Child Adolesc. Psychiatry 53, 780-789.
Sobel, L.J., Bansal, R., Maia, T.V., Sanchez, J., Mazzone, L., Durkin, K., Liu, J., Hao, X., Ivanov, I., Miller, A., Greenhill, L.L., Peterson, B.S., 2010w. Basal ganglia surface morphology and the effects of stimulant medications in youth with attention deficit hyperactivity disorder. Am. J. Psychiatry 167, 977-986.

Spencer, R.C., Klein, R.M., Berridge, C.W., 2012. Psychostimulants act within the prefrontal cortex to improve cognitive function. Biol. Psychiatry 72, 221-227.

Suskauer, S.J., Simmonds, D.J., Fotedar, S., Blankner, J.G., Pekar, J.J., Denckla, M.B., Mostofsky, S.H., 2008. Functional magnetic resonance imaging evidence for abnormalities in response selection in attention deficit hyperactivity disorder: differences in activation associated with response inhibition but not habitual motor response. J. Cogn. Neurosci. 20, 478-493.

Villemonteix, T., Purper-Ouakil, D., Romo, L. 2014. Is emotional dysregulation a component of attention deficit/hyperactivity disorder. Encephale.

Volkow, N.D., 2012w. Long-term safety of stimulant use for ADHD: findings from nonhuman primates. Neuropsychopharmacology 37, 2551-2552.

Voormolen, E.H.J., Wei, C., Chow, E.W.C., Bassett, A.S., Mikulis, D. J., Crawley, A.P., 2010. Voxel-based morphometry and automated lobar volumetry: the trade-off between spatial scale and statistical correction. Neuroimage 49, 587-596.

Warren, B.L., Iniguez, S.D., Alcantara, L.F., Wright, K.N., Parise, E. M., Weakley, S.K., Bolanos-Guzman, C.A., 2011w. Juvenile administration of concomitant methylphenidate and fluoxetine alters behavioral reactivity to reward- and mood-related stimuli and disrupts ventral tegmental area gene expression in adulthood. J. Neurosci. 31, 10347-10358.

Wechsler, D., 1999. Wechsler Abbreviated Scale of Intelligence ed. The Psychological Corporation, San Antonio.

Winkler, A.M., Kochunov, P., Blangero, J., Almasy, L., Zilles, K., Fox, P.T., Duggirala, R., Glahn, D.C., 2009. Cortical thickness or grey matter volume? The importance of selecting the phenotype for imaging genetics studies. Neuroimage 53, 1135-1146.

Zuvekas, S.H., Vitiello, B., 2012w. Stimulant medication use in children: a 12-year perspective. Am. J. Psychiatry 169, 160-166. 